\title{
QUADROTOR UAV CONTROL FOR TRANSPORTATION OF CABLE SUSPENDED PAYLOAD
}

\author{
Tom Kusznir, Jarosław Smoczek \\ AGH University of Science and Technology \\ Faculty of Mechanical Engineering and Robotics \\ Mickiewicza Av. 30, 30-059 Krakow, Poland \\ tel.: +48126173104 \\ e-mail: tkusznir@agh.edu.pl,smoczek@agh.edu.pl
}

\begin{abstract}
Payload transportation with UAV's (Unmanned Aerial Vehicles) has become a topic of interest in research with possibilities for a wide range of applications such as transporting emergency equipment to otherwise inaccessible areas. In general, the problem of transporting cable suspended loads lies in the under actuation, which causes oscillations during horizontal transport of the payload. Excessive oscillations increase both the time required to accurately position the payload and may be detrimental to the objects in the workspace or the payload itself. In this article, we present a method to control a quadrotor with a cable suspended payload. While the quadrotor itself is a nonlinear system, the problem of payload transportation with a quadrotor adds additional complexities due to both input coupling and additional under actuation of the system. For simplicity, we fix the quadrotor to a planar motion, giving it a total of 4 degrees of freedom. The quadrotor with the cable suspended payload is modelled using the Euler-Lagrange equations of motion and then partitioned into translation and attitude dynamics. The design methodology is based on simplifying the system by using a variable transformation to decouple the inputs, after which sliding mode control is used for the translational and pendulum dynamics while a feedback linearizing controller is used for the rotational dynamics of the quadrotor. The sliding mode parameters are chosen so stability is guaranteed within a certain region of attraction. Lastly, the results of the numerical simulations created in MATLAB/Simulink are presented to verify the effectiveness of the proposed control strategy.
\end{abstract}

Keywords: transport, UAV, quadrotor, under actuated, cable suspended payload, sliding mode control

\section{Introduction}

Vertical take-off landing (VTOL) unmanned aerial vehicles have found themselves in an increasing number of applications in both commercial and military application in recent years. Civil applications of UAV's include rescue operations, cartographic and geodetic research, agriculture, construction, and filming [1,3. The concept of using VTOL aircraft such as helicopters has long been used for transportation of cable-suspended loads such as in the case of heli-logging or during rescue operations. Due to the dangers during certain rescue scenarios such as during fires or storms, it is preferable to use UAVs where it is either too risky or impossible to use manned VTOL aircraft. This, along with the interest by commercial companies to find new methods of transporting products has led to an increase in interest of solving the problem of UAV payload transportation.

While there are many different VTOL UAV architectures, the most common is the quadrotor due to its mechanical simplicity. The quadrotor is a highly nonlinear and an inherently unstable system, and this should be considered when designing a controller. There are two approaches to developing a control system for the quadrotor, a linear controller, or a nonlinear controller. In general, the linear controllers can easily be optimized such as in [9] using LQR, though it comes at disadvantage of only being optimal, or even stable near the linearized points. Due to this if, a quadrotor must perform some more aggressive manoeuvres, which deviate from these linearized points a nonlinear control strategy would be preferred.

Several methods of transporting a payload by a quadrotor have been applied [11], one of which 
is with a robotic arm and gripper. This method generally keeps the payload close to the centre of mass of the quadrotor, slowing down the rate at which the quadrotor can rotate, thus decreasing the manoeuvrability of the quadrotor [5]. Using a UAV to transport a payload with an attached manipulator has been done in [2]. This decrease in manoeuvrability can be a major drawback in certain cases. The other method is by suspending the payload on a rope, in this case the payload behaves like a pendulum. While it does not have the drawback of decreased manoeuvrability as in the previous case, it does cause payload oscillation. The oscillations of the payload pose a hazard to the surrounding environment, the payload itself, and to the UAV by causing instability. Excessive oscillations also increase the time to deliver the payload to its desired position while also reducing the accuracy of reaching the desired target. To avoid this control strategies, need to be employed that not only position the quadrotor itself but also damp the payload oscillations which may be dangerous to people or objects in the workspace, the payload itself and even the quadrotor.

In the case of a quadrotor transporting, a cable suspended load or swung load several methods were used such as dynamic programming to generate a trajectory that minimizes the payload swing [10]. In [5] the authors used mathematical program with complimentary constraints (MPCC) to optimize the trajectory that satisfy certain constraints. In [12] the authors used a feedback modelbased control system to stabilize the quadrotor while using feedforward input shaping technique to reduce dampen the payload oscillation. A feedback-linearizing controller was applied to the quadrotor with input shaping used to reducing payload oscillation in [13]. In [8] geometric control was used to control the quadrotor carrying a payload while in [4] geometric control was utilized for lifting the payload from a stationary position. Another interesting approach to solve the problem was using Interconnection Damping Assignment-Passivity Based Control (IDA-PBC) to control the quadrotor [6] and to attenuate the payload oscillation in [7].

The rest of the article is organized as follows. Section two provides the mathematical description of the quadrotor dynamics developed using the Euler-Lagrange method. The control strategy, which consist of using feedback linearization and sliding mode control (SMC), are discussed in Section three. Numerical results of the simulation are presented in Section four. The concluding argument is made in Section five.

\section{Dynamic model}

The system under consideration is a planar model of a quadrotor UAV with a cable-suspended payload presented in Fig. 1. The system is modelled under assumption that the unactuated pendulum is a point-mass suspended at the end of a massless rigid rod. The symbols in Fig. 1 are described as the UAV quadrotor mass $M$, pendulum mass $m$, rope length $l$, pitch and payload swing angle $\theta$ and $\alpha$.

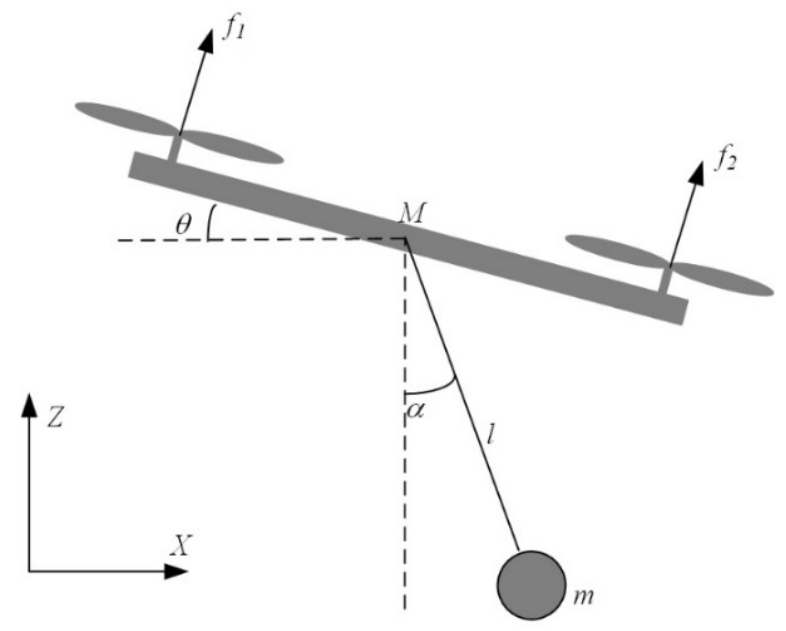

Fig. 1. Quadrotor transporting a payload in the X-Z plane 
The system's motion equations are derived using the Euler-Lagrange's equation (1) for the generalized coordinates $q=[x, z, \alpha, \theta]^{T}$ and input vector $[f, \tau]^{T}$ with the total thrust $f=f_{1}+f_{2}$ and the torque $\tau$ provided by the rotors:

$$
\frac{d}{d t}\left(\frac{\partial L}{\partial \dot{q}}\right)-\frac{\partial L}{\partial q}=[f, \tau]^{T}
$$

where the Lagrangian function $L(q, \dot{q})=K(q, \dot{q})-U(q)$ is determined based on the kinetic $K$ and potential $U$ energy of the system:

$$
\begin{aligned}
K & =\frac{1}{2} M \dot{x}^{2}+\frac{1}{2} M \dot{z}^{2}+\frac{1}{2} J_{\theta} \dot{\theta}^{2}+\frac{1}{2} m \dot{x}_{p}{ }^{2}+\frac{1}{2} m \dot{z}_{p}{ }^{2}, \\
U & =M g z+m g z_{c}
\end{aligned}
$$

where $x, z, x_{p}=x+l \sin \alpha, z_{p}=z-l \cos \alpha$ are the quadrotor and payload position in the $X-Z$ plane, $J_{\theta}$ is the inertia moment of the quadrotor and $g$ is gravitational acceleration.

The motion equations derived from (1) are as follows:

$$
\begin{aligned}
& f \sin \theta=(M+m) \ddot{x}+m l \cos \alpha \ddot{\alpha}-m l \dot{\alpha}^{2} \sin \alpha \\
& f \cos \theta=(M+m) \ddot{z}+m l \sin \alpha \ddot{\alpha}+m l \dot{\alpha}^{2} \cos \alpha+(M+m) g \\
& 0=m l^{2} \ddot{\alpha}+m l \ddot{x} \cos \alpha+m l \ddot{z} \sin \alpha+m l g \sin \alpha \\
& \tau=J_{\theta} \ddot{\theta}
\end{aligned}
$$

The equations (3) can be further rewritten to:

$$
\begin{aligned}
& \ddot{x}=\frac{M+m \cos ^{2} \alpha}{M(M+m)} f \sin \theta+\frac{m \sin \alpha \cos \alpha}{M(M+m)} f \cos \theta+\frac{m l \dot{\alpha}^{2} \sin \alpha}{M+m} \\
& \ddot{z}=\frac{m \sin \alpha \cos \alpha}{M(M+m)} f \sin \theta+\frac{M+m \sin ^{2} \alpha}{M(M+m)} f \cos \theta-\frac{m l \dot{\alpha}^{2} \cos \alpha}{M+m}-g \\
& \ddot{\alpha}=-\frac{\cos \alpha}{M l} f \sin \theta-\frac{\sin \alpha}{M l} f \cos \theta \\
& \ddot{\theta}=\frac{\tau}{J_{\theta}}
\end{aligned}
$$

\section{Control strategy}

The control objective is to move the quadrotor from the initial position $(x, z)$ to the desired position $\left(x_{d}, z_{d}\right)$ with suppressing the pendulum oscillation. The proposed control approach assumes the two control subsystems for the altitude control and longitude control of the quadrotor with cablesuspended payload, respectively. The control strategy is developed under assumption that the pitch angle of the quadrotor is restricted within $-\pi / 2<\theta<\pi / 2$ and the control input $f$ is decomposed to the $f_{x}$ and $f_{z}$ acting the quadrotor horizontal and vertical motion, respectively. This implies redefinition of the control input $f$ as follows:

$$
\begin{aligned}
& f=\frac{f_{z}}{\cos \theta_{d}} \\
& \left|\theta_{d}\right|=\left|\operatorname{arctg}\left(\frac{f_{x}}{f_{z}}\right)\right|<\frac{\pi}{2},
\end{aligned}
$$


where:

$f_{x}=f \sin \theta$,

$f_{z}=f \cos \theta$.

\subsection{Altitude and attitude control}

The feedback linearization procedure is applied to develop the altitude and attitude control of a quadrotor. From (4), the vertical position control can be derived to:

$$
f_{z}=\frac{M\left((M+m)\left(\ddot{e}_{z}+g\right)+m l \dot{\alpha}^{2} \cos \alpha-F_{x} m \sin \alpha \cos \alpha\right)}{M+m \sin ^{2} \alpha},
$$

where $e_{z}=z-z_{d}$.

In order to track given trajectories, the $\ddot{e}$ was chosen as:

$$
\ddot{e}_{z}=-k_{p z} e_{z}-k_{d z} \dot{e}_{z},
$$

where $k_{p z}$ and $k_{d z}$ are positive constants.

The similar approach can be applied for pitch angle control by choosing the control signal $\tau$ as:

$$
\tau=-J_{\theta}\left(k_{p \theta} e_{\theta}+k_{d \theta} \dot{e}_{\theta}-\ddot{\theta}_{d}\right),
$$

where $e_{\theta}=\theta-\theta_{d}$.

\subsection{Horizontal position of the quadrotor with pendulum control}

For the horizontal motion of a quadrotor with pendulum, a dynamic model can be expressed as:

$$
\begin{aligned}
& \dot{x}_{1}=x_{2} \\
& \dot{x}_{2}=f_{1}(x)+b_{1}(x) f_{x}, \\
& \dot{x}_{3}=x_{4} \\
& \dot{x}_{4}=f_{2}(x)+b_{2}(x) f_{x}
\end{aligned}
$$

where:

$$
\begin{aligned}
& x_{1}=x-x_{d}, \\
& x_{3}=\alpha, \\
& f_{1}(x)=\frac{m \sin \alpha \cos \alpha}{M(M+m)} f_{z}+\frac{m l \dot{\alpha}^{2} \sin \alpha}{M+m}, \\
& b_{1}(x)=\frac{M+m \cos ^{2} \alpha}{M(M+m)}, \\
& f_{2}(x)=-\frac{\sin \alpha}{M l} f_{z}, \\
& b_{2}(x)=-\frac{\cos \alpha}{M l},
\end{aligned}
$$

and $f_{z}$ is considered as the time varying parameter.

The sliding surface $s$ is proposed as

$$
s=c_{1} x_{1}+c_{2} x_{2}+c_{3} x_{3}+c_{4} x_{4},
$$

where $c_{1}, c_{2}, c_{3}, c_{4}$ are constants. 
Splitting the control signal $f_{x}$ into equivalent $u_{e q}$ and switching control $u_{s w}$ :

$$
f_{x}=u_{e q}+u_{s w}
$$

The equivalent control is derived from the time derivative of $s$ :

$$
\dot{s}=c_{1} \dot{x}_{1}+c_{2}\left(f_{1}+b_{1} u_{e q}\right)+c_{3} \dot{x}_{3}+c_{4}\left(f_{2}+b_{2} u_{e q}\right)=0,
$$

that results in:

$$
u_{e q}=-\frac{c_{1} x_{2}+c_{2} f_{1}+c_{3} x_{4}+c_{4} f_{2}}{c_{2} b_{1}+c_{4} b_{2}} .
$$

In deriving the switching control law the Lyapunov candidate function is defined as $V=s^{2} / 2$. Differentiating $V$ with respect to time and substituting (13), we have:

$$
\begin{aligned}
& \dot{V}=s \dot{s}=s\left[c_{1} \dot{x}_{1}+c_{2} f_{1}+c_{3} \dot{x}_{3}+c_{4} f_{2}+\left(c_{2} b_{1}+c_{4} b_{2}\right)\left(u_{e q}+u_{s w}\right)\right] . \\
& =s\left(c_{2} b_{1}+c_{4} b_{2}\right) u_{s w}
\end{aligned}
$$

Choosing the switching control as:

$$
u_{s w}=-\frac{k_{1} s+k_{2} \operatorname{sgn}(s)}{c_{2} b_{1}+c_{4} b_{2}},
$$

which leads to:

$$
\dot{V}=-k_{1} s^{2}-k_{2}|s| \leq 0,
$$

where $k_{1}$ and $k_{2}$ are positive constants.

Assuming $f_{x}=u_{e q}$, selecting parameter $c_{2}$ as $c_{2}=1$, and linearizing the closed-loop system (9) on an equilibrium point $x_{0}=[0,0,0,0]^{T}$, the closed-loop (9) can be expressed as:

$$
\begin{aligned}
& \dot{x}_{2}=f_{1}(x)+b_{1}(x) u_{e q}=\frac{c_{4} f_{z}}{(M+m)\left(l-c_{4}\right)} x_{3}-\frac{l}{l-c_{4}}\left(c_{1} x_{2}+c_{3} x_{4}\right) \\
& \dot{x}_{4}=f_{2}(x)+b_{2}(x) u_{e q}=-\frac{f_{z}}{(M+m)\left(l-c_{4}\right)} x_{3}+\frac{1}{l-c_{4}}\left(c_{1} x_{2}+c_{3} x_{4}\right)
\end{aligned} .
$$

To achieve asymptotically convergence of state trajectories the parameters $c_{1}, c_{3}, c_{4}$ should be chosen such that $A$ is Hurwitz. Locating the closed-loop eigenvalues for example at $\lambda=-\sqrt{g / l}$ the parameters of sliding mode control are derived from:

$$
\operatorname{det}(s I-A)=\prod_{i=1}^{n=3}\left(s-\lambda_{i}\right),
$$

where:

$$
\begin{aligned}
& \operatorname{det}(s I-A)=s^{3}+\frac{c_{1} l-c_{3}}{l-c_{4}} s^{2}+\frac{f_{z}}{(M+m)\left(l-c_{4}\right)} s+\frac{c_{1} f_{z}}{(M+m)\left(l-c_{4}\right)}, \\
& \prod_{i=1}^{n=3}\left(s-\lambda_{i}\right)=s^{3}+p_{2} s^{2}+p_{1} s+p_{0},
\end{aligned}
$$

that finally results in following sliding mode control parameters: 


$$
\begin{aligned}
& c_{1}=\frac{p_{0}}{p_{1}}, \\
& c_{2}=1, \\
& c_{3}=l c_{1}-\frac{p_{2}}{p_{1}(M+m)} f_{z} \\
& c_{4}=l-\frac{f_{z}}{p_{1}(M+m)} .
\end{aligned}
$$

The system will be asymptotically stable as long as $f_{z}=f \cos \theta>0$, which is satisfied as the quadrotor's pitch angle is restricted within $-\pi / 2<\theta<\pi / 2$.

\section{Simulation results}

The simulations were carried out for quadrotor parameters $M=2 \mathrm{~kg}$ and $J_{\theta}=0.1 \mathrm{kgm}^{2}$. The parameters of the altitude (7) and attitude (8) controllers were chosen empirically as $k_{p z}=k_{d z}=4$, $k_{p \theta}=200, k_{d \theta}=20$. The sliding mode controller parameters were selected as $k_{1}=1, k_{2}=0.01$. The control system performances were tested for unit-step input function. The simulations were carried out for different quadrotor-pendulum system parameters: the rope length $l=\{1,2\} \mathrm{m}$ and payload mass $m=\{0.2,0.6\} \mathrm{kg}$.

Figures 2-5 presents the simulations for pendulum length $l=1 \mathrm{~m}$ and mass $m=0.6 \mathrm{~kg}$, while Fig. 6-9 depict the results conducted for $l=2 \mathrm{~m}$ and $m=0.2 \mathrm{~kg}$. The simulation results prove that using the proposed control strategy the pendulum oscillations are effectively suppressed at different operating conditions. Since the eigenvalues of the closed-loop are located at $\lambda=-\sqrt{g / l}$ the quadrotor-pendulum achieves the desired position $\left(x_{d}=1 \mathrm{~m}\right)$ without overshoot (Fig. 2 and 6). The lack of overshoot is also observed for vertical position of the quadrotor. For these location of the closed-loop eigenvalues, the settling time and maximum value of the sway angle do not depend on the pendulum mass, and depend on the rope length, e.g. the increase of rope length causes the increase in settling time of the quadrotor-pendulum horizontal position (5.2 and 6.0 seconds for the rope length 1 and 2 meters, respectively) and the decrease in maximum sway angle ( $0.058 \mathrm{rad}$ and $0.039 \mathrm{rad}$ for $l=1 \mathrm{~m}$ and $l=2 \mathrm{~m}$, respectively). Thus, the specific performance criteria can be met by choosing proper location of closed-loop poles and tuning the altitude and attitude control parameters.

\section{Conclusions}

In this article, we developed a control for transporting a cable suspended payload on a quadrotor in planar motion. By splitting the system into two subsystems and using sliding mode control for the positional control and a feedback-linearizing controller for the rotational control, we were able to obtain satisfactory results when moving to the desired position. The controller also suppresses oscillations effectively when the physical parameters are changed, which proves the robustness of the proposed control strategy. These have been proved by numerical simulations carried out in MATLAB/Simulink.

Future research includes parameter optimization to minimize the settling time of transporting the payload to the desired position while keeping the oscillation within a specified safety bound.

\section{Acknowledgement}

The work has been supported by the Polish Ministry of Science and Higher Education from funds for year 2019. 


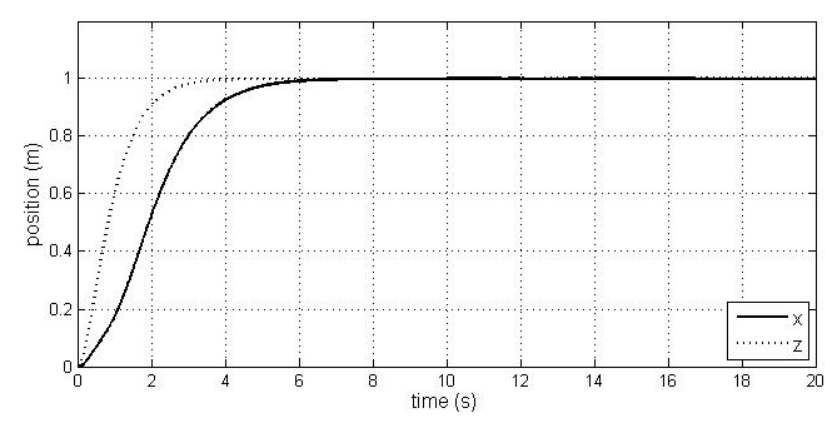

Fig. 2. Position in $X-Z$ plane: $l=1 \mathrm{~m}, \mathrm{~m}=0.6 \mathrm{~kg}$

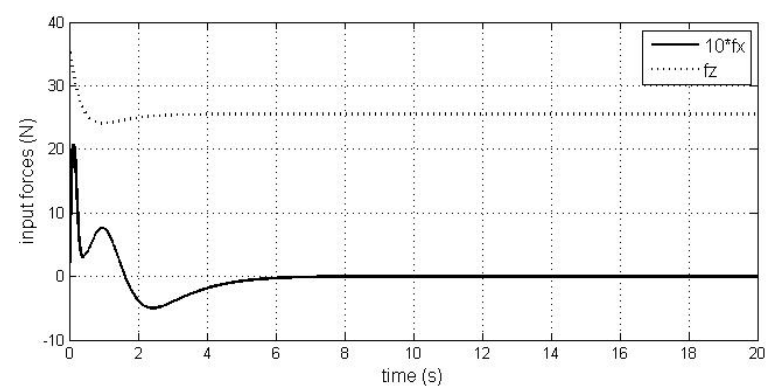

Fig. 4. Input forces $10 * f_{x}$ and $f_{z}: l=1 \mathrm{~m}, \mathrm{~m}=0.6 \mathrm{~kg}$

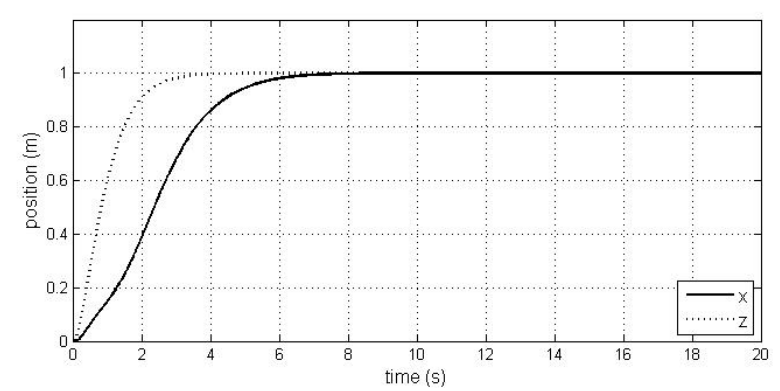

Fig. 6. Position in $X-Z$ plane: $l=2 \mathrm{~m}, \mathrm{~m}=0.2 \mathrm{~kg}$

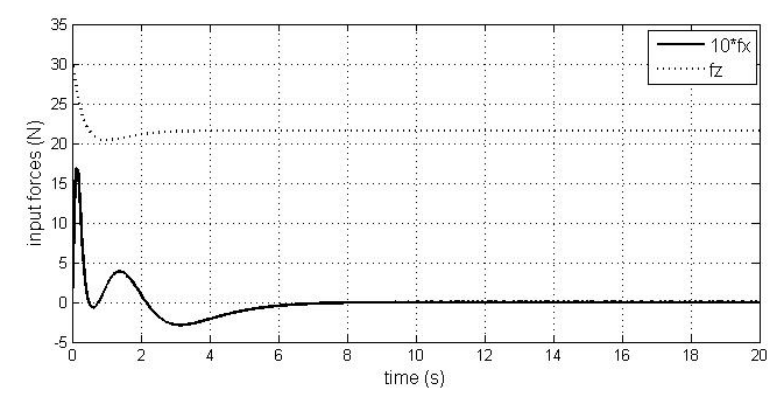

Fig. 8. Input forces $10^{*} f_{x}$ and $f_{z}: l=2 \mathrm{~m}, \mathrm{~m}=0.2 \mathrm{~kg}$

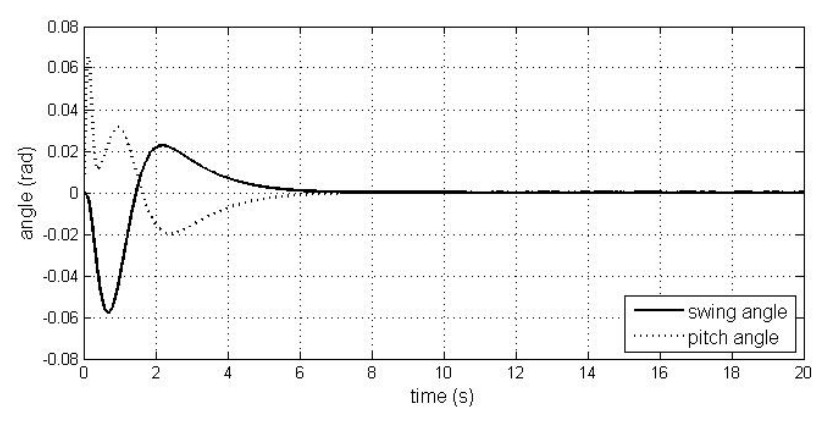

Fig. 3. Swing and pitch angle: $l=1 \mathrm{~m}, \mathrm{~m}=0.6 \mathrm{~kg}$

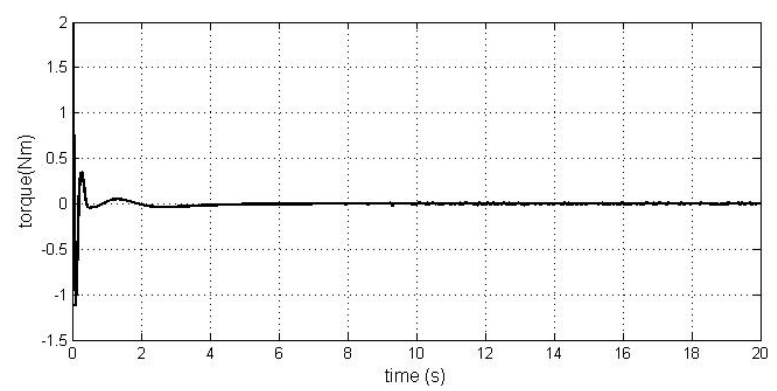

Fig. 5. Torque: $l=1 \mathrm{~m}, \mathrm{~m}=0.6 \mathrm{~kg}$

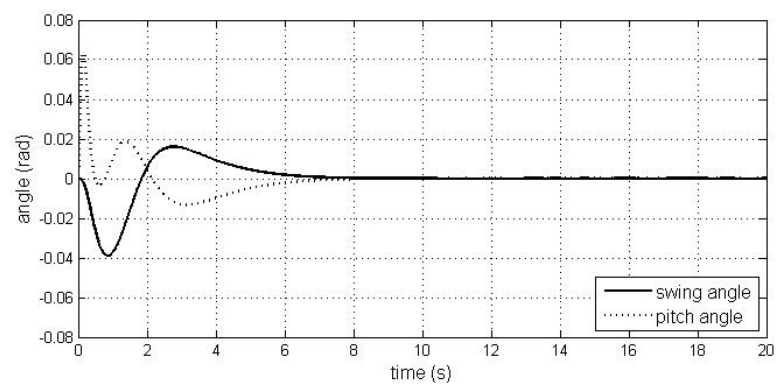

Fig. 7. Swing and pitch angle: $l=2 \mathrm{~m}, \mathrm{~m}=0.2 \mathrm{~kg}$

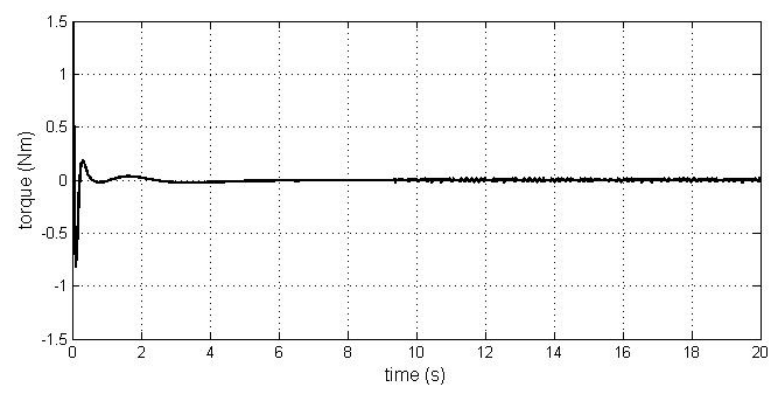

Fig. 9. Torque: $l=2 \mathrm{~m}, \mathrm{~m}=0.2 \mathrm{~kg}$

\section{References}

[1] Andrzejewski-Popow, Ł., Kotas, T., The future development of unmanned air vehicles, Journal of KONES Powertrain and Transport, Vol. 22, No. 4, 2015.

[2] Chmaj, G., Buratowski, T., Uhl, T., Seweryn, K., Banaszkiewicz, M., The dynamics influence of the attached manipulator on unmanned aerial vehicle, Aerospace Robotics, GeoPlanet Earth and Planetary Sciences, Springer-Verlag Berlin Heidelberg, pp. 109-119, 2013.

[3] Ciopcia, M., Szczepański, C., Concept of tiltrotor UAV control system, Journal of KONES Powertrain and Transport, Vol. 24, No. 1, 2017. 
[4] Cruz, P. J., Oishi, M., Fierro, R., Lift of a Cable-suspended load by a quadrotor: A Hybrid System Approach, IEEE American Control Conference (ACC), pp. 1887-1892, Chicago 2015.

[5] Foehn, P., Falanga, D., Kuppuswamy, N., Tedrake, R., Scaramuzza, D., Fast trajectory optimization for agile quadrotor manoeuvres with a cable-suspended payload, Robotics: Science and Systems, Cambridge, MA, USA 2017.

[6] Guerrero, M.E., Mercado, D. A., Lozano, R., Garcia, C. D., IDA-PBC methodology for a quadrotor UAV transporting a cable-suspended payload., International Conference on Unmanned Aircraft Systems (ICUAS), pp. 470-476, Denver 2015.

[7] Guerrero-Sánchez, M. E., Mercado-Ravell, D. A., Lozano R., García-Beltrán C. D., Swingattenuation for a quadrotor transporting a cable-suspended payload, ISA Transactions, 2017.

[8] Lee, T., Sreenath, K., Kumar V., Geometric control of cooperating multiple quadrotor UAVs with a suspended load, Proceedings of the IEEE Conference on Decision and Control, pp. 55105515, Florence 2013.

[9] Ołdziej, D., Gosiewski, Z., Modelling of dynamic and control of six-rotor autonomous unmanned aerial vehicle, Solid State Phenomena, Vol. 198, pp. 220-225, 2013.

[10] Palunko, I., Fierro, R., Cruz, P., Trajectory generation for swing free manoeuvres of a quadrotor with suspended payload: A dynamic programming approach, IEEE International Conference on Robotics and Automation, pp. 2691-2697, 2012.

[11] Ruggiero, F., Lippiello, V., Ollero, A., Aerial manipulation: A literature review, IEEE Robotics and Automation Letters, Vol. 3, No. 3, pp. 1957-1964, 2018.

[12] Sadr, S., Moosavian, S. Ali A., Zarafshan, P., Dynamics Modeling and Control of a Quadrotor with Swing Load, Journal of Robotics, 2014.

[13] Zúñiga, N. S., Muñoz, F., Márquez, M. A., Quesada, E. S. E., Carrillo, L. R. G., Load transportation using single and multiple quadrotor aerial vehicles with swing load attenuation International Conference on Unmanned Aircraft Systems (ICUAS), pp. 269-278, Dallas TX, USA 2018. 